\title{
Synchronous lymph node metastasis in apparently low-risk T1 colon cancer
}

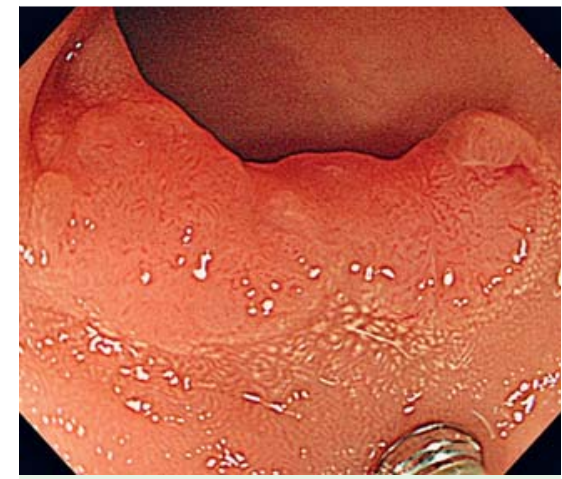

Fig. 1 Endoscopic image of the sigmoid colon of an 81-year-old woman undergoing treatment for colon cancer shows a 15 -mm flatelevated lesion with a slight depression.

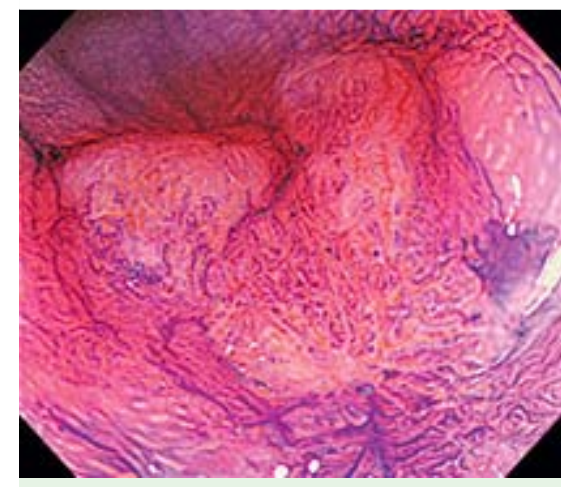

Fig. 3 Chromoendoscopy with magnification following crystal violet staining shows a severe and irregular pattern in the pit.

The risk for lymph node metastasis is low in T1 colorectal cancer when none of the following pathologic findings is present: invasion to a depth of $1000 \mu \mathrm{m}$ or more, positive horizontal or vertical margin, lymphovascular permeation, severe tumor budding, and either a poorly differentiated or a mucinous component [ $1-3]$. To the best of our knowledge, no case of such a low-risk T1 tumor associated with lymph node metastasis has been reported.

An 81-year-old woman was referred to our hospital for the treatment of colon cancer. In the sigmoid colon, colonoscopy identified a $15-\mathrm{mm}$ flat-elevated lesion with a slight depression ( $\bullet$ Fig.1). On

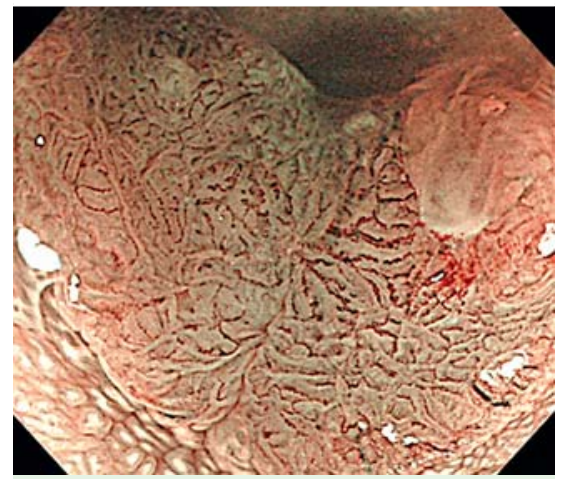

Fig. 2 Magnifying endoscopy with narrowband imaging shows the dilated, tortuous microvascular architecture of the tumor, which lacks uniformity in the depression.

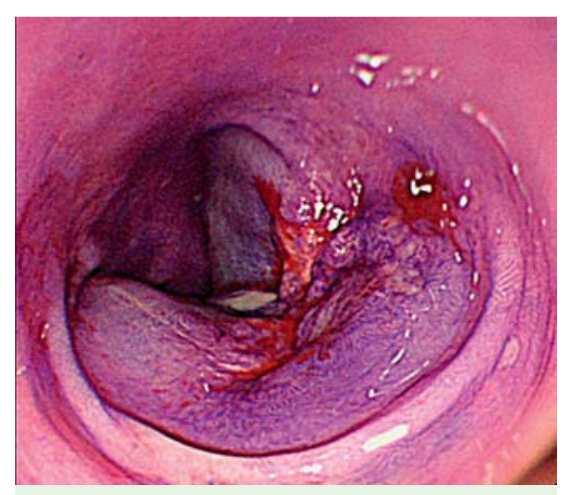

Fig.4 A distinct nonlifting sign is observed after a diagnostic submucosal injection.

narrow-band imaging, the microvascular architecture of the tumor was dilated and tortuous, and it lacked uniformity in the depression ( Fig.2). Chromoendoscopy with magnification following crystal violet staining revealed a severe and irregular pattern in the pit ( Fig.3). The lesion was diagnosed as a deeply invasive submucosal adenocarcinoma; therefore, we performed a diagnostic submucosal injection, and a distinct nonlifting sign was recognized ( $\bullet$ Fig.4). For this reason, the patient underwent sigmoidectomy with lymph node dissection. Pathologic evaluation of the resected specimen confirmed that the tumor had invaded the shallow part of the submucosal layer (invasion depth $560 \mu \mathrm{m}$ from the muscularis mucosae; Fig.5). This lesion was considered to be a low risk $\mathrm{T} 1$ tumor because of the lack of pathologic findings associated with lymph node invasion. Nonetheless, pathologic examination of the resected lymph nodes showed that cancer cells had metastasized to a regional node (๑ Fig.6).

The current treatment strategy after endoscopic resection for a $\mathrm{T} 1$ tumor depends on the perceived metastatic risk based on the pathologic findings [1]. The long-term outcomes of $\mathrm{T} 1$ colon cancer after endoscopic resection are reportedly acceptable $[4,5]$. However, a standard surveillance strategy after endoscopic resection has not yet been established. This case may suggest the need for imaging surveillance to detect lymph node metastases after the endoscopic resection of apparently low risk T1 tumors.

Endoscopy_UCTN_Code_CCL_1AD_2AB

Competing interests: None

\section{Masao Yoshida, Kenichiro Imai, Kinichi Hotta, Hiroyuki Ono}

Division of Endoscopy, Shizuoka Cancer Center, Shizuoka, Japan

\section{References}

1 Watanabe T, Itabashi $M$, Shimada $Y$ et al. Japanese Society for Cancer of the Colon and Rectum (JSCCR) guidelines 2010 for the treatment of colorectal cancer. Int J Clin Oncol 2012; 17: 1-29

2 Kitajima K, Fujimori T, Fujii $S$ et al. Correlations between lymph node metastasis and depth of submucosal invasion in submucosal invasive colorectal carcinoma: a Japanese collaborative study. J Gastroenterol 2004; 39: 534- 543

3 Bosch S, Teerenstra S, de Wilt JHW et al. Predicting lymph node metastasis in pT1 colorectal cancer: a systematic review of risk factors providing rationale for therapy decisions. Endoscopy 2013; 45: 827-834

4 Yoda Y, Ikematsu H, Matsuda T et al. A largescale multicenter study of long-term outcomes after endoscopic resection for submucosal invasive colorectal cancer. Endoscopy 2013; 45: 718-724

5 Yoshii S, Nojima M, Nosho $K$ et al. Factors associated with risk for colorectal cancer recurrence after endoscopic resection of $\mathrm{T} 1$ tumors. Clin Gastroenterol Hepatol 2014; 12: $292-302$. e3 

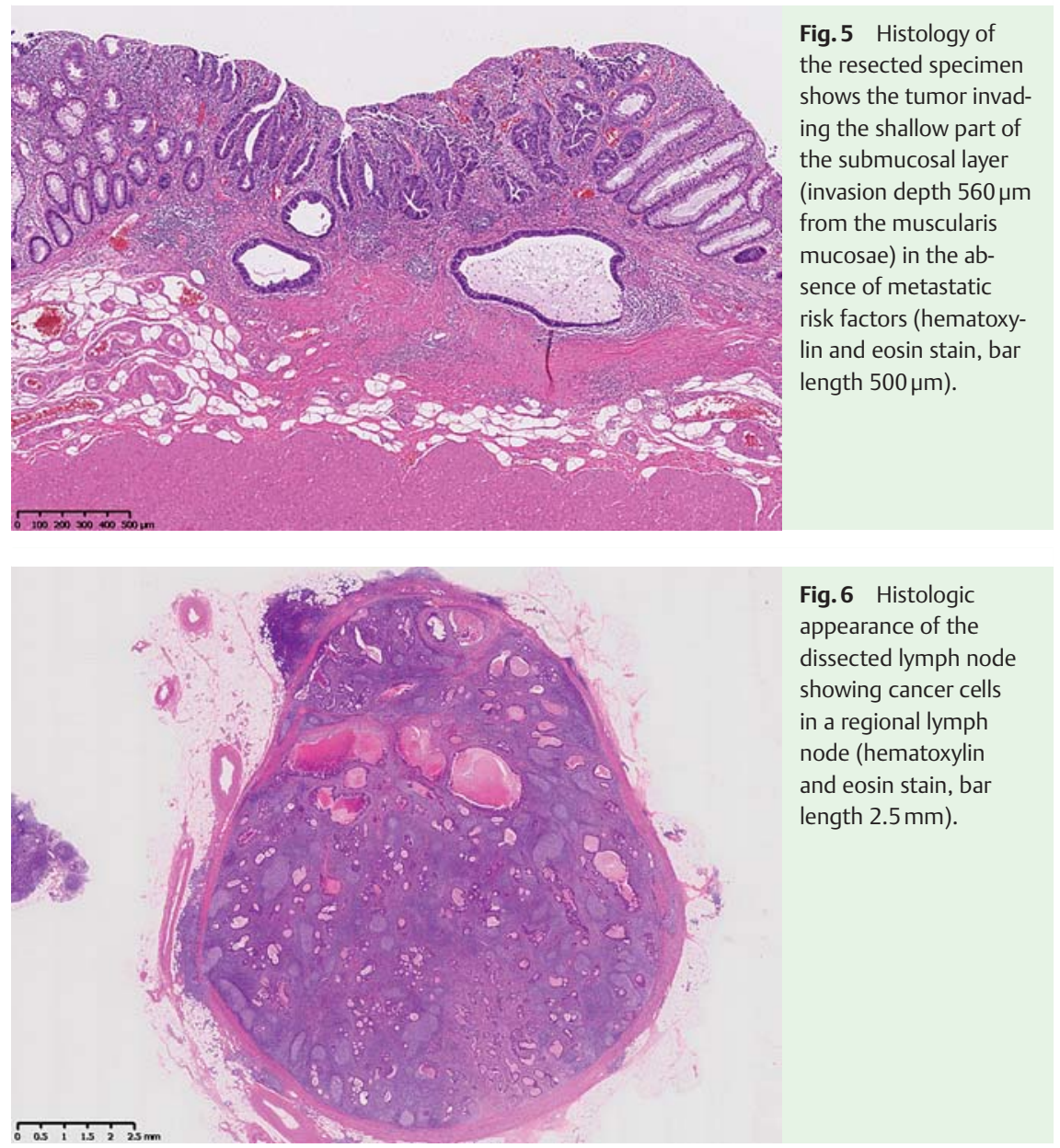

\section{Bibliography}

Dol http://dx.doi.org/

10.1055/s-0034-1377593

Endoscopy 2014; 46: E526-E527

(C) Georg Thieme Verlag KG

Stuttgart · New York

ISSN 0013-726X

\section{Corresponding author}

Masao Yoshida, MD

Division of Endoscopy, Shizuoka Cancer Center 1007 Shimonagakubo, Nagaizumi, Sunto-gun Shizuoka 411-8777

Japan

Fax: +81-55-9895783

ma.yoshida@scchr.jp 